\title{
Tachistoscopic number estimation in patients with unilateral cerebral lesions
}

\author{
ELIZABETH K. WARRINGTON AND MERLE JAMES
}

From the National Hospital, Queen Square, London

It has been shown that lesions of the right temporal lobe result in a variety of perceptual deficits (Milner, 1958; Kimura, 1963), which have been interpreted in terms of right hemisphere dominance for certain non-verbal functions. Warrington and James (1967), however, have shown that the right parietal lobe may be more important than the right temporal lobe in certain kinds of visuo-perceptual function. Furthermore, they present some evidence of differentiation of function within the right hemisphere: tasks of visual retention for spatial information and recognition of incomplete contour information were differentially impaired.

Recently there have been several tachistoscopic studies in which it has been possible to make more precise measurements of perceptual recognition. Kimura (1963) found, with a number estimation task, that the right temporal group was impaired relative to the left. Dorf, Mirsky, and Mishkin (1965) compared tachistoscopic recognition of groups of letters in the right and left visual fields in normal subjects and in right and left temporal lobectomy cases, and found that in both patient groups the contralateral field was impaired relative to the ipsilateral field. A contralateral quadrantic defect may be present after temporal lobectomy. Though Dorf et al. (1964) report a higher incidence of field defects in the right temporal groups, they found that the severity of the recognition defect was unrelated to the presence of a field defect. Meier and French (1965) found impaired discrimination of pairs of fragmented concentric circle patterns in patients with right temporal lobectomy compared with left temporal lobectomy. No direct measure of simple visual discrimination, such as a visual form threshold, was made in any of these investigations.

Holmes (1918) first described the clinical syndrome of visual disorientation, and he drew attention to the patient's inability to count an array of similar objects either by vision alone or by pointing to each in turn. A characteristic feature of this disability was that the patient as often overestimated as under- estimated the number of objects, which led Holmes to interpret the disability as secondary to visual disorientation in space rather than as a manifestation of unilateral visual neglect. McFie, Piercy, and Zangwill (1950) used a similar test in their clinical investigation of patients with right parietal lesions, and found that ability to count scattered coins or matchsticks was commonly impaired.

The task of tachistoscopic number estimation is in some ways similar to the test used in clinical neurology. The subject is required to count (but not identify) scattered dots or any other simple visual forms under conditions of presentation where eye movements are not possible.

In the present study two tachistoscopic measurements were made: number estimation and the detection threshold for perception of dots and letters, the latter being a measure of a defective visual field. A task more closely analogous to the counting of scattered objects as administered clinically was also included. The aim of this investigation was to assess the relationship between a simple detection measurement and performance on a more complex perceptual task, and elucidate further the role of the right hemisphere in spatial perceptual functions.

\section{METHOD}

PATIENT GROUPS Subjects were selected from patients referred to the Psychology Department of The National Hospital, Queen Square, for testing. Every patient with good evidence, obtained radiologically or at operation, of a unilateral cerebral lesion, able to cooperate in the test situation, and right-handed for writing, was included in the series.

Forty-nine patients selected in this way, and seen consecutively, were tested. Twenty-nine patients had right hemisphere lesions and 20 had left hemisphere lesions. Dr. Gilford, of the Radiological Department, classified all patients as having frontal, temporal, parietal or occipital lobe lesions, or a combination of these four categories. Patients with lesions involving more than one lobe were included in both groups for comparisons between lobes. Three of the 20 left hemisphere cases had lesions involving more than one lobe, 
two of them having lesions involving three lobes. Thirteen of these patients had clinical evidence of dysphasia. Of the right hemisphere group, six of 29 had lesions extending over two lobes; none had lesions involving three lobes. The left hemisphere group included 12 patients with temporal lobe involvement and 10 with parietal lobe involvement (two patients fell in both groups), while in the right hemisphere group there were 15 patients with temporal lobe lesions and 11 with parietal lobe involvement (two patients appeared in both groups). Thirteen of the left hemisphere patients and 15 of the right hemisphere patients had neoplasms. Age, sex, pathology, locus of lesions, and presence of field defects and dysphasia are given in Table I.

Fifteen patients with peripheral nerve lesions were tested as controls.

APPARATUS A Dodge type tachistoscope, with a variable exposure duration from $2 \mathrm{msec}$. to $1,600 \mathrm{msec}$., according to a logarithmic scale, was used, giving time exposure series $2.0,2.5,3.2,4.0,5.0,6.4,8.0,10.0,12.5$, and $16 \mathrm{msec}$. Multiplying factors of 10 and 100 were used to extend the range. The background and exposure field were both $6 \times 4$ in. subtending an angle of $14^{\circ}$ at the retina $\left(7^{\circ}\right.$ either side of the fixation point), and were matched for brightness and colour. (Further details are given by Kinsbourne and Warrington, 1962.)

TEST STIMULI Three sets of test stimuli were prepared and drawn in black ink on white cards 6 in. $\times 4$ in.
1 Single dot stimuli Three single dot test stimuli $\frac{1}{2} \mathrm{~cm}$ in diameter subtending a visual angle of $\frac{1}{2}^{\circ}$, arranged in the centre, $2 \frac{1}{2}^{\circ}$ to the right and $2 \frac{1}{2}^{\circ}$ to the left, were used.

2 Single letter stimuli Each letter was $1 \mathrm{~cm}$. in height, subtending a visual angle of $1^{\circ}$. Three arrangements of single letter stimuli were used; centre of the visual field, $2 \frac{1}{2}^{\circ}$ to the right of centre, and $2 \frac{1}{2}^{\circ}$ to the left of centre. (All letters of the alphabet were used.)

3 Groups of dots Three to seven dots were used as test stimuli. Three spatial arrangements of the groups of dots, central, to the right of the centre of the field, and to the left of the centre of the field, were used. The arrays of dots subtended a maximum of $3^{\circ}$ of visual angle in the horizontal plane and $5^{\circ}$ in the vertical plane. There were three test stimuli for each number of dots, 15 for each visual field, making 45 test stimuli in all; for example, for four dots, three dots arranged centrally, three arranged to the right, and three arranged to the left of the centre of the tachistoscopic field.

TACHISTOSCOPIC PROCEDURE FOR DETECTION MEASUREMENTS Single dot stimuli and single letter stimuli were used to determine the tachistoscopic detection times in terms of the minimum duration of exposure required to report the presence or absence of the dot stimuli, or the recognition of the letter stimuli. The dot detection time was determined by the shortest duration of exposure at which five successive stimuli were reported correctly in each of the three positions. Cards with right, left, and

TABLE I

CLINICAL DETAILS OF PATIENT GROUPS

\begin{tabular}{|c|c|c|c|c|c|c|c|c|c|c|c|c|}
\hline \multicolumn{7}{|c|}{ Left Hemipshere } & \multicolumn{6}{|c|}{ Right Hemisphere } \\
\hline $\begin{array}{l}\text { Case } \\
\text { No. }\end{array}$ & Age & Sex & Pathology & Locus & Dysphasia & Field Defects & $\begin{array}{l}\text { Case } \\
\text { No. }\end{array}$ & Age & Sex & Pathology & Locus & Field Defects \\
\hline 1 & 35 & $\mathbf{F}$ & $\mathbf{N}$ & $\mathbf{T}$ & + & RHH & 1 & 17 & $\mathbf{F}$ & $\mathbf{A}$ & $\mathrm{T}$ & LHH \\
\hline 3 & 66 & $\mathbf{F}$ & $\mathbf{N}$ & $\mathbf{P}$ & & & 2 & 53 & $\mathbf{M}$ & $\mathbf{N}$ & $\mathrm{T}$ & LHH \\
\hline 6 & 39 & $\mathbf{M}$ & $\mathbf{N}$ & $\mathbf{T}$ & + & & 3 & 44 & $\mathbf{F}$ & $\mathbf{V}$ & $\mathbf{T}$ & LHH \\
\hline 8 & 38 & $\mathbf{M}$ & $\mathbf{A}$ & $\mathbf{T}$ & + & & 4 & 44 & $\mathbf{F}$ & $\mathbf{N}$ & $\mathbf{F}$ & \\
\hline 10 & 35 & $\mathbf{M}$ & $\mathbf{A}$ & $\mathbf{P}$ & + & & 6 & 64 & $\mathbf{F}$ & $\mathbf{N}$ & $\mathrm{T}$ & \\
\hline 11 & 31 & $\mathbf{M}$ & $\mathbf{N}$ & $\mathbf{P}$ & & & 8 & 25 & $\mathbf{F}$ & $\mathbf{N}$ & $\mathbf{T}$ & LUQ \\
\hline 13 & 33 & $\mathbf{M}$ & $\mathbf{N}$ & $\mathrm{T}$ & + & RHH & 10 & 41 & $\mathbf{M}$ & V & $\mathrm{T}$ & LUQ \\
\hline 15 & 19 & $\mathbf{F}$ & A & $T$ & & & 12 & 43 & $\mathbf{M}$ & $\mathbf{N}$ & $F$ & \\
\hline 17 & 45 & $\mathbf{M}$ & $\mathbf{N}$ & $\mathbf{F}, \mathbf{P}$ & & & 13 & 21 & $\mathbf{F}$ & $\mathbf{L}$ & $\mathbf{T}$ & \\
\hline 19 & 17 & $\mathbf{M}$ & A & $\mathbf{P}$ & & & 15 & 26 & $\mathbf{F}$ & V & PT & \\
\hline 10 & 60 & $\mathbf{M}$ & $\mathbf{N}$ & $\mathbf{P}$ & + & & 16 & 25 & $\mathbf{M}$ & $\mathbf{A}$ & $T$ & \\
\hline 21 & 57 & $\mathbf{M}$ & $\mathbf{N}$ & $\mathbf{T}$ & + & & 19 & 51 & $\mathbf{M}$ & $\mathbf{L}$ & $\mathbf{F}$ & LUQ \\
\hline 23 & 33 & $\mathbf{F}$ & $\mathbf{N}$ & $\mathrm{T}$ & + & & 21 & 54 & $\mathbf{M}$ & $\mathbf{N}$ & PO & LHH \\
\hline 24. & 53 & $\mathbf{M}$ & $\mathbf{N}$ & $\mathrm{T}$ & + & RHH & 23 & 46 & $\mathrm{~F}$ & $\mathbf{N}$ & $T$ & LHH \\
\hline 25 & 30 & $\mathbf{F}$ & $\mathbf{V}$ & $\mathbf{P}$ & + & & 24 & 57 & $\mathbf{M}$ & $\mathbf{N}$ & $\mathbf{F}$ & LHH \\
\hline 20 & 73 & $\mathbf{M}$ & $\mathbf{N}$ & $T$ & + & RHH & 25 & 31 & $\mathrm{~F}$ & L & $\mathrm{T}$ & LUQ \\
\hline 32 & 62 & $\mathbf{F}$ & $\mathbf{N}$ & $\mathbf{P}$ & & & 26 & 25 & $\mathbf{M}$ & $\bar{L}$ & $\mathrm{~T}$ & \\
\hline 35 & 25 & $\mathbf{M}$ & V & F,P,T, & + & & 27 & 52 & $F$ & $\mathbf{N}$ & $T$ & \\
\hline 36 & 38 & $\mathbf{F}$ & $\mathbf{N}$ & $\mathrm{T}$ & & & 28 & 34 & $\mathbf{M}$ & $\mathbf{N}$ & $\mathbf{P}$ & \\
\hline 37 & 31 & $\mathbf{M}$ & $\mathbf{M}$ & F,P,T & + & & 29 & 42 & $\mathrm{~F}$ & $\mathbf{L}$ & $T$ & LUQ \\
\hline & & & & & & & 30 & 61 & $\mathrm{~F}$ & $\mathbf{L}$ & $\mathbf{F}$ & LUQ \\
\hline & & & & & & & 33 & 63 & $\mathbf{M}$ & $\mathbf{N}$ & $\mathbf{P}$ & LUQ \\
\hline & & & & & & & 34 & 68 & $\mathbf{M}$ & $\mathbf{N}$ & FP & \\
\hline & & & & & & & 35 & 69 & $\mathbf{F}$ & $\mathbf{N}$ & $\mathbf{P}$ & LHH \\
\hline & & & & & & & 36 & 59 & $\mathbf{F}$ & $\mathbf{N}$ & PO & LHH \\
\hline & & & & & & & 37 & 56 & $\mathbf{F}$ & V & TP & \\
\hline & & & & & & & 38 & 62 & $\mathbf{M}$ & V & $\mathbf{P}$ & \\
\hline & & & & & & & 39 & 61 & $\mathbf{F}$ & $\mathbf{N}$ & PO & \\
\hline & & & & & & & 40 & 60 & $\mathbf{M}$ & $\mathbf{N}$ & $\mathbf{P}$ & \\
\hline $\begin{array}{l}A= \\
+ \text { pre }\end{array}$ & cess of & $\begin{array}{r}\mathbf{N} \\
\text { yspha }\end{array}$ & $\begin{array}{l}\text { neoplasm } \\
\text { ia }\end{array}$ & $\mathbf{V}=$ & cular disorder & $\begin{array}{l}\mathbf{L}=\text { lobec } \\
\mathbf{M}=\text { miss }\end{array}$ & $\begin{array}{l}\text { omy } \\
\text { le wor }\end{array}$ & & & & & \\
\hline $\mathbf{F}=\mathbf{f}$ & tal & $\mathbf{P}$ & parietal & $\mathbf{T}=$ & poral & $=$ occipital & & & & & & \\
\hline
\end{tabular}


centre dots, and an equal number of blank cards, were presented in random order.

The single letter detection time was similarly determined, except that no blank cards were given. Single letters in each of the three positions-central, right, and left-were presented in random order. The minimum exposure duration at which five successive stimuli were correctly reported (in each of the three positions) was recorded as the single letter detection time.

TACHISTOSCOPIC PROCEDURE FOR NUMBER ESTIMATION A constant exposure duration of $100 \mathrm{msec}$. was used throughout this experiment. The 45 test stimuli were shown in a random order, so that there was no expectation as to the part of the visual field in which the dot stimuli would appear. Each test stimulus was used once.

TEST OF DOT COUNTING A random arrangement of 15 dots and 15 dashes was drawn on a card 10 in. $\times 9$ in., either in a straight line or in a series of loops. The patient was required to count the total number of dots and dashes on each card. The cards were rotated through $180^{\circ}$ and the patient again counted the number of dots and dashes. No time limit was imposed. The patient control group did not do this test.

\section{RESULTS}

DETECTION MEASUREMENTS The detection measurements for the single dot stimuli and the single letter stimuli in each of the three positions were trans- formed to an arithmetical scale, the switch settings having values increasing by one. After transformation the measurements for single dots and single letters were summed for each position, giving a composite threshold value for the central, right, and left visual fields. The mean detection measurement values for each group are given in Table II.

The detection measurements of the right and left hemisphere groups obtained in the three positions in the visual field were compared with those of the control group. The Mann-Whitney $U$ test was used throughout. The $\mathrm{z}$ and $\mathrm{U}$ values and significance level for each group comparison are given in Table III. The temporal and parietal subgroups have been compared with the control group measurements in each of the three positions, and the right and left parietal subgroups have been compared with the right and left non-parietal cases respectively. The left hemisphere group was found to be impaired relative to the control group in the ipsilateral and contralateral fields, but not in the central field, while the right hemisphere group was impaired relative to the control group in all three field positions. No impairment of the detection measurement was found in the left temporal subgroup, and only in the ipsilateral field of the left parietal group. The right temporal group had impaired detection measurements in all three fields, while the right parieta

TABLE II

MEAN DETECTION TIMES AND MEAN ERRORS IN NUMBER ESTIMATION FOR EACH PATIENT GROUP

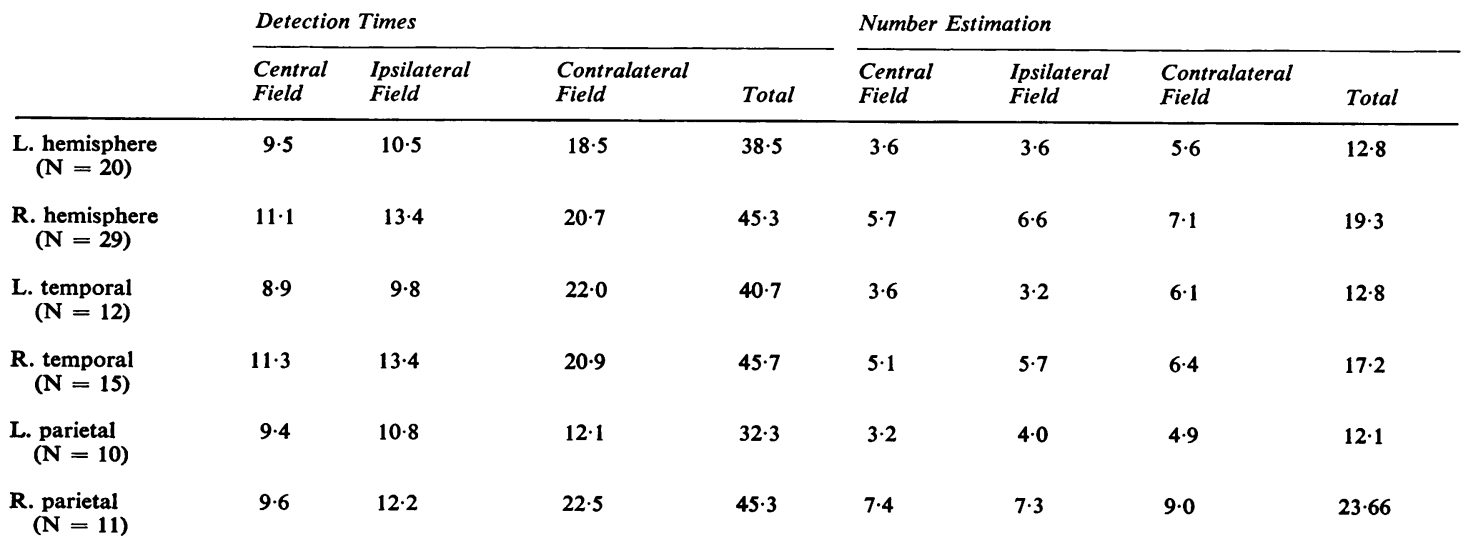

TABLE IIa

MEAN DETECTION TIMES AND MEAN ERRORS IN NUMBER ESTIMATION FOR CONTROL GROUP

Detection Times

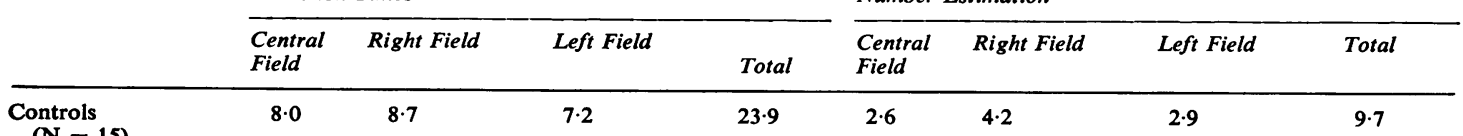


group was impaired in the ipsilateral and contralateral fields, but not in the central field. Neither the right nor the left parietal groups were impaired relative to the non-parietal cases.

NUMBER ESTIMATION The number of errors in the central, contralateral, and ipsilateral fields of vision was recorded. The mean error scores for the right and left hemisphere cases and the parietal and temporal subgroups are given in Table II. As in the above analysis, the Mann-Whitney $U$ test has been used in all group comparisons, and the $z$ and $U$ values and significance level for each group comparison are given in Table III. The right hemisphere cases were significantly worse than the control patients in all three visual fields. No significant difference was found between the left hemisphere and the control group in any of the three visual fields. The right temporal cases were significantly worse than the control cases only in the contralateral field, and the right parietal cases were significantly worse than the control cases in the central field, ipsilateral field, and contralateral field. Though the right parietal group obtained a higher mean error score than the right temporal group, there were no significant differences between the right parietal cases and the right non-parietal cases except in the contralateral visual field. Neither the left temporal nor the left parietal groups showed any impairment relative to the control group.

When one compares the results of detection measurements with number estimation no consistent relationship is observed. Although number estimation was not impaired in any field of the left hemisphere group as compared with controls, certain of the detection measurements do show a deficit. Though the right hemisphere group as a whole was impaired on both tasks, the right temporal group was as impaired as the right parietal group on the detection measurements (more impaired in the central field of vision), yet only the right parietal group showed a deficit on number estimation in the central and ipsilateral visual fields.

CORRELATIONS BETWEEN TACHISTOSCOPIC TESTS Spearman's rank correlation was calculated to assess the degree of correlation between the detection measurements and the number estimation scores in each of the three visual fields for each group of patients. The correlations, together with the level of significance, are given in Table IV. In the control group, very low correlations, none of them significant, were obtained. In contrast, the performance on these two tasks was significantly correlated in the left hemisphere group in all three visual fields, while in the right hemisphere group a significant correlation was obtained only in the left visual field (contralateral to the lesion). Thus there is a dissociation between these two measures in the control group and right hemisphere group (except in the contralateral visual field), but not in the left hemisphere group, which indicates that impaired detection may have affected the performance on the number estimation task in the left hemisphere group, but is not of critical importance in determining the deficit shown by the right hemisphere group on this task.

ANALYSIS OF ERRORS Errors of over and underestimation on the number estimation task were recorded. The mean ratio of errors of overestimation to the total error score is given in Table

TABLE III

GROUP COMPARISONS ON DETECTION MEASUREMENTS AND NUMBER ESTIMATION

\begin{tabular}{|c|c|c|c|c|c|c|}
\hline \multirow{4}{*}{ Left hemisphere v. controls } & \multicolumn{3}{|c|}{ Detection Measurements } & \multicolumn{3}{|c|}{ Number Estimation } \\
\hline & Central & Ipsilateral & Contralateral & \multirow{2}{*}{$\begin{array}{l}\text { Central } \\
Z=85.0\end{array}$} & Ipsilateral & Contralateral \\
\hline & $Z=0.93$ & $Z=2 \cdot 33^{1}$ & $Z=2.43^{1}$ & & $Z=1.05$ & $Z=1.08$ \\
\hline & $p>0.05$ & $\mathrm{p}<0.02$ & $\mathrm{p}<0.02$ & $p>0.05$ & $p>0.05$ & $\bar{p}>0.05$ \\
\hline Right hemisphere v. controls & $Z=2 \cdot 29^{1}$ & $Z=3 \cdot 1^{2}$ & $Z=4 \cdot 8^{3}$ & $Z=2.63^{2}$ & $Z=2 \cdot 24^{1}$ & $Z=3.75^{3}$ \\
\hline & $\mathrm{p}<0.03$ & $\mathrm{p}<0.002$ & $p<0.0001$ & $\bar{p}<0.01$ & $\bar{p}<0.03$ & $\bar{p}<0.001$ \\
\hline Left temporal v. controls & $U=66 \cdot 0$ & $U=60 \cdot 0$ & $\mathbf{U}=50 \cdot 0$ & $U=79 \cdot 5$ & $\mathrm{U}=\mathbf{8 4 \cdot 5}$ & $\mathrm{U}=69 \cdot 5$ \\
\hline & $p>0.05$ & $p>0.05$ & $p>0.05$ & $p>0.05$ & $p>0.05$ & $\mathrm{p}>0.05$ \\
\hline Right temporal v. controls & $U=51 \cdot 5^{1}$ & $\mathrm{U}=53 \cdot 5^{1}$ & $U=10 \cdot 0^{3}$ & $U=65.0$ & $U=78 \cdot 0$ & $\mathrm{U}=40 \cdot 0^{1}$ \\
\hline & $\mathrm{p}<0.02$ & $\mathrm{p}<0.02$ & $\mathrm{p}<0.002$ & $p>0.05$ & $p>0.05$ & $p<0.02$ \\
\hline Left parietal v. controls & $U=61 \cdot 0$ & $U=35 \cdot 5^{1}$ & $U=41 \cdot 0$ & $U=61 \cdot 0$ & $U=49 \cdot 0$ & $\mathbf{U}=64 \cdot 0$ \\
\hline & $p>0.05$ & $p<0.05$ & $p>0.05$ & $p>0.05$ & $p>0.05$ & $p>0.05$ \\
\hline Right parietal v. controls & $U=47 \cdot 0$ & $U=35 \cdot 0^{1}$ & $U=8 \cdot 5^{2}$ & $U=22 \cdot 5^{2}$ & $U=41 \cdot 0^{1}$ & $U=10 \cdot 0^{2}$ \\
\hline & $p>0.05$ & $p<0.02$ & $p<0.002$ & $\mathrm{p}<0.002$ & $p<0.05$ & $\mathrm{p}<0.002$ \\
\hline Left parietal v. left non-parietal & $U=49 \cdot 0$ & $U=46 \cdot 0$ & $\mathrm{U}=37 \cdot 0$ & $\mathrm{U}=50 \cdot 0$ & $\mathrm{U}=53.0$ & $\mathbf{U}=40 \cdot 0$ \\
\hline & $\begin{array}{l}p>0.05 \\
U=92.0\end{array}$ & $\begin{array}{l}p>0.05 \\
U=83.0\end{array}$ & $\begin{array}{l}p>0.05 \\
U=97.0\end{array}$ & $\begin{array}{l}p>0.05 \\
U=59.0\end{array}$ & $\begin{array}{l}p>0.05 \\
U=81.0\end{array}$ & $p>0.05$ \\
\hline Right parietal v. right non-parietal & $\begin{array}{l}U=92.0 \\
p>0.05\end{array}$ & $p>0.05$ & $p>0.05$ & $p>0.05$ & $\begin{array}{l}U=81.0 \\
p>0.05\end{array}$ & $p<0.05$ \\
\hline
\end{tabular}

'Significant at $5 \%$ level

Significant $1 \%$ level

SSignificant $0 \cdot 1 \%$ level 
TABLE IV

CORRELATION BETWEEN DETECTION MEASUREMENT AND NUMBER ESTIMATION IN THE CENTRAL, RIGHT, AND LEFT VISUAL FIELDS

\begin{tabular}{lccc} 
& Central Field & Left Field & Right Field \\
\hline $\begin{array}{l}\text { Controls } \\
\mathbf{N}=15\end{array}$ & 0.175 & 0.015 & 0.271 \\
$\begin{array}{l}\text { Left hemisphere } \\
\mathbf{N}=20\end{array}$ & $0.727^{3}$ & $0.459^{1}$ & $0.502^{1}$ \\
$\begin{array}{l}\text { Right hemisphere } \\
\mathbf{N}=29\end{array}$ & 0.206 & $0.484^{2}$ & 0.008
\end{tabular}

1 Significant at the $5 \%$ level

2 Significant at the $1 \%$ level

- Significant at the $0 \cdot 1 \%$ level

$\mathrm{V}$ for each patient group in each of the visual fields. As the data were to some extent bimodally distributed the median test of significance was used in these group comparisons, In the central visual field the right hemisphere group had a significantly higher ratio of errors of overestimation than did the control group, while the left hemisphere group did not differ significantly from the control group. In the right visual field the left hemisphere group made significantly fewer errors of overestimation than the control group, while the other comparisons with the

\section{TABLE V}

MEAN RATIO OF ERRORS OF OVERESTIMATION TO TOTAL ERROR SCORE IN THE THREE PATIENT GROUPS

\begin{tabular}{llll} 
Patient Group & $\begin{array}{l}\text { Visual Field } \\
\text { Central }\end{array}$ & Right & Left \\
\hline Controls & 0.23 & 0.53 & 0.39 \\
Left hemisphere & 0.25 & 0.25 & 0.28 \\
Right hemisphere & 0.35 & 0.60 & 0.32
\end{tabular}

control group did not reach significance. However, in a direct comparison between both the contralateral and ipsilateral fields in the left and right hemisphere groups, the right hemisphere group obtained significantly higher ratios of overestimation errors.

DOT CounTING Four trials were given: each was scored pass (if accurate) or fail (if inaccurate). Errors of over-and underestimation were recorded. Sixteen right hemisphere cases and 11 left hemisphere cases failed two or more trials. Using the $\chi^{2}$ test of significance, there was no association between laterality of lesion and failure on dot counting $\left(\chi^{2}=0 \cdot 17\right)$. However, using the same method of scoring, the right parietal cases (excluding cases common to both groups) were significantly worse than the right temporal cases $\left(\chi^{2}=8.56\right)$ while no difference was found between the left parietal and left temporal cases $\left(\chi^{2}=0.06\right)$. Also, the left hemi- sphere dysphasics were not more impaired on this task than the left hemisphere non-dysphasics.

The median test of significance was used to assess the association between performance on the tachistoscopic number estimation task (total error score for three visual fields) and dot counting. In the right hemisphere group there was a significant association at the $5 \%$ level of probability $\left(\chi^{2}=4.50\right)$ but not in the left hemisphere group $\left(\chi^{2}=0.04\right)$. Therefore, though the left hemisphere group is as impaired as the right hemisphere group on dot counting, only in the right hemisphere group is failure associated with locus of lesion (parietal) and with performance on the number estimation task.

Nine right-sided cases and five left-sided cases made one or more errors of overestimation. This difference in incidence of errors of overestimation is not significant $\left(\chi^{2}=0.54\right)$.

\section{DISCUSSION}

In this study the performance of right and left hemisphere groups was compared on two tachistoscopic tests, namely detection measurements and number estimation. One clinical test of counting an array of dots and dashes was included. A further analysis based on localization within each hemispere was carried out.

Kimura (1966) has shown for a group of normal right-handed subjects that number estimation of dots is more accurate in the left visual field than the right visual field (a centrally arranged display was not used). In patients, the ipsilateral field of the left hemisphere group (left field) would be superior to the ipsilateral field of the right hemisphere group (right field) if the normal bias were maintained. Therefore, in the present study, no direct comparisons between patient groups were attempted; except for those comparisons within the hemisphere, the patient groups were compared with the appropriate control measurements. The right field of each patient group was compared with the right field of the control group, and similarly with the left field.

The detection measurements show that there is a deficit in both ipsilateral and contralateral fields for both right and left hemisphere groups relative to the control group. The central field of the left hemisphere group was not found to be significantly different from normal. The mean detection measurements in the right hemisphere group were worse in all three visual fields than the corresponding left hemisphere measurements. On the number estimation task the left hemisphere group did not differ significantly from the control group, while the right hemisphere group showed a deficit in all three 
visual fields. A consideration of the subgroup comparisons indicates that these findings cannot simply be interpreted as showing that the number estimation task is less discriminative, performance on this being impaired only if the detection measurements are sufficiently poor. Within the right hemisphere group there is a dissociation between performance on the detection measurements and number estimation. The right temporal group shows a deficit in all three visual fields on the detection task, but not a significant impairment on the number estimation task in the ipsilateral and central fields, while the converse is true of the right parietal group, where in the central visual field there was no deficit on the detection task, but a significant impairment on the number estimation task. Further, though the right parietal group was not impaired relative to the right non-parietal cases on detection measurements, in the contralateral visual field it was impaired on number estimation. Thus no one-to-one relationship was found between detection measurements and number estimation.

Correlations between the detection measurements and number estimation in each of the three visual fields for each group of patients were calculated. While in the left hemisphere group performance on these two tasks was significantly correlated in all three visual fields, no significant correlations were found in the control group, nor in the central and ipsilateral fields in the right hemisphere group. There was, however, a significant correlation in the contralateral field of the right hemisphere group. These findings may be interpreted to show that though field defects, as indicated by impaired detection measurements, may have been responsible for the impaired number estimation in the left hemisphere group, they cannot account for the deficit in number estimation found in the right hemisphere group (except in the contralateral field).

Kimura (1963) reports that right temporal cases are impaired relative to left temporal cases on a number estimation task similar to that used in the present investigation. This finding has not been confirmed in this investigation except in the contralateral visual field, where there was also a deficit on the detection measurement. However, the right parietal group show an impairment in all three visual fields, though the deficit is not socircumscribed as to show a significant difference between the right parietal and the right non-parietal groups.

On the dot counting test the right hemisphere group was not impaired relative to the left hemisphere group, though the right parietal group was significantly worse than the right non-parietal group. In spite of this failure to find an association between dot counting and laterality of lesion, there was an association between performance on the tachistoscopic number estimation task and dot counting in the right hemisphere group, but not in the left hemisphere group. This finding suggests that the dot counting task is complex, and that there may be more than one basis for failure on it.

An error in the number estimation task may be either an overestimation or an underestimation. Holmes (1918) observed that errors of overestimation were common; the patient would count the same item twice or more, apparently not aware that it had already been included in the count. While unilateral neglect phenomena might account for failure to count all of a number of scattered objects, errors of overestimation cannot be explained in this way. In the present study, the right hemisphere group produced the highest proportion of errors of overestimation on the tachistoscopic number estimation task, though this was not observed in the clinical test of counting dots and dashes. Patients in the right hemisphere group, as well as being quantitatively worse on this task, show a qualitative feature also observed clinically when a patient counts and overestimates an array of scattered objects. In the tachistoscopic test, the exposure duration of $100 \mathrm{msec}$. is too short to permit voluntary eye movements. Therefore the difficulty observed clinically in counting scattered objects cannot be accounted for in terms of disordered eye movement and poor oculomotor coordination, or as secondary to unilateral neglect of space.

The span of apprehension for the number of items in an array was classically regarded as the fixed number which could be immediately processed. However, experimental investigations have shown that not only is the span determined by parameters such as exposure duration and light intensity (Woodworth and Schlosberg, 1955) but also that the verbal response time increases in a continuous function with arrays of two and more items (Saltzman and Garner, 1948). That is, it takes longer to respond 'three' to three items than 'two' to two items. If one accepts that number estimation is a 'serial' process even with very small numbers of items, and that the task is achieved by a rapid scanning (which may or may not involve counting) of the visual image or immediate memory of the image, then it is reasonable to suggest that impaired spatial perception would result in difficulty on this task. In scanning serially an array of dots which has no other means of identification, the spatial position becomes of critical importance. A deficit in processing spatial information might then account for impaired number estimation.

It is of some interest to consider these findings in relation to two neurological syndromes, visual 
disorientation and visual spatial agnosia. Visual disorientation was defined by Holmes as the inability to localize the position and distance of objects in space by sight alone. He described a number of other disabilities which he regarded as secondary to the primary disorder, including disordered eye movements, impairment of reading a connected passage, and counting scattered objects. He interpreted these disabilities in terms of a disturbance of the local sign functions of the retina (Holmes, 1919). Grossly impaired tachistoscopic number estimation has been recorded in one case of visual disorientation (Godwin-Austen, 1965). McFie et al. (1950) have reported eight cases of visuo-spatial agnosia, with unilateral right parietal lesions. Five of these patients made errors in counting scattered objects, yet none had visual disorientation. There must, therefore, be a deficit other than defective localization of objects in space to account for these findings. While in visual disorientation the cardinal feature is a failure to localize a single object, in visuo-spatial agnosia it has been suggested that the basic deficit is that of integrating spatial information in a visuo-motor task (Warrington et al., 1966). Another important distinction is that visual disorientation can occur in either half visual field, contralateral to the lesion (Cole, Schutta, and Warrington, 1962), or in both visual fields with a bilateral occipital lesion (Holmes, 1918). Visuospatial disorders from a unilateral lesion of the right parietal region are not restricted to the contralateral visual field or the contralateral limb. It is therefore suggested that areas within the occipital lobes contribute to the absolute localization of an object by integrating the retinal sign cue with other kinaesthetic information on the position of the eyes and head. However, the integration of temporal or successive spatial stimuli which determines the spatial relations of two or more objects is impaired by unilateral lesions of the right hemisphere, but not of the left hemisphere.

\section{SUMMARY}

Forty-nine patients with unilateral cortical lesions were tested by two tachistoscopic procedures, detection measurement and number estimation, and one test of dot counting. Right and left hemisphere groups were compared. Both right and left hemisphere groups were impaired relative to the control group on the detection task, and the right hemi- sphere group was impaired on the number estimation task. The right parietal group, but not the right temporal group, was significantly worse than the control group on number estimation. There was no correlation between performance on the detection task and number estimation in the central or ipsilateral field of the right hemisphere group. Performance on the dot counting task was not related to laterality of lesion. The findings are discussed in relation to visuo-spatial agnosia and visual disorientation.

We wish to thank the physicians and surgeons of The National Hospital for permission to study and report our findings on patients under their care, and Dr. J. Bull for his cooperation in this investigation. We are grateful to Drs. G. Ettlinger and R. T. C. Pratt for their helpful advice and comments in the preparation of this manuscript. Our thanks are particularly due to Dr. E. S. Gilford of the Department of Radiology, The National Hospital, for his examination and classification of the radiological data. One of us (M. J.) was supported by a grant from the Medical Research Council.

\section{REFERENCES}

Cole, M., Shutta, M. S., and Warrington, E. K. (1962). Visual disorientation in homonymous half-fields. Neurology (Minneap.), 12, 257-263.

Dorff, J. E., Mirsky, A. F., and Mishkin, M. (1965). Effects of unilateral temporal lobe removals in man on tachistoscopic recognition in the left and right visual fields. Neuropsychologia, 3, 39-51.

Godwin-Austen, R. B. (1965). A case of visual disorientation. $J$. Neurol. Neurosurg. Psychiat., 28, 453-458.

Holmes, G. (1918). Disturbances of visual orientation. Brit. J. Ophthal. 2, 449-468, 506-516.

- (1919). Disturbances of visual space perception. Lecture II. Brit. med.J., 2, 230-233.

Kimura, D. (1963). Right temporal-lobe damage: Perception of unfamiliar stimuli after damage. Arch. Neurol. (Chic.), 8, 264-271.

- (1966). Dual functional asymmetry of the brain in visual perception. Neuropsychologia, 4, 275-285.

Kinsbourne, M., and Warrington, E. K. (1962). The effect of an aftercoming random pattern on the perception of brief visual stimuli. Quart. J. exp. Psychol., 14, 223-234.

McFie, J., Piercy, M. F., and Zangwill, O. L. (1950). Visual spatial agnosia associated with lesions of the right cerebral hemisphere. Brain, 73, 167-190.

Meier, M. J., and French, L. A. (1965). Lateralized deficits in complex visual discrimination and bilateral transfer of reminiscence following unilateral temporal lobectomy. Neuropsychologia, 3, 261-272.

Milner, B. (1958). Psychological defects produced by temporal lobe excision. Res. Publ. Ass. Res. nerv. ment. Dis., 36, 244-257

Saltzman, I. J., and Garner, W. R. (1948). Reaction time as a measure of span of attention. J. Psychol., 25, 227-241.

Warrington, E. K., and James, M. (1967). Disorders of visual perception in relation to right hemisphere lesions. Neuropsychologia, in the press.

,$- \frac{8}{-}$ and Kinsborne, M. (1966). Drawing disability in relation to laterality of cerebral lesion. Brain, 89, 53-82.

Woodworth, R. S., and Schlosberg, H. (1955). Experimental Psycho$\log y$, 3rd., ed., ch. 4. 72-106 Methuen, London. 